\title{
Variable induction of PRDM1 and differentiation in chronic lymphocytic leukemia is associated with anergy
}

\author{
Andrew Duckworth, ${ }^{1}$ Mark Glenn, ${ }^{1}$ Joseph R. Slupsky, ${ }^{1}$ Graham Packham, ${ }^{2}$ and Nagesh Kalakonda ${ }^{1}$ \\ ${ }^{1}$ Haematology, Department of Molecular and Clinical Cancer Medicine, Institute of Translational Medicine, University of Liverpool, Liverpool, United \\ Kingdom; and ${ }^{2}$ Cancer Sciences, Faculty of Medicine, University of Southampton, Southampton, United Kingdom
}

\section{Key Points}

- Differentiation of CLL cells in response to IL-21 and cytosine guanine dinucleotide-enriched oligo-deoxynucleotides (CpG-ODN) is variable and linked to PRDM1 induction.

- The failure of CLL cells to express or induce PRDM1 correlates with anergy.

Despite antigen engagement and intact B-cell-receptor (BCR) signaling, chronic lymphocytic leukemia (CLL) cells fail to undergo terminal differentiation. We hypothesized that such failure may be due to anergy, as CLL cells exhibit variable levels of nonresponsiveness to surface IgM stimulation that is reversible in vitro. Moreover, anergy is associated with reduced differentiation capacity in normal B cells. We investigated responses of CLL cells to two potent differentiation-promoting agents, IL-21 and cytosine guanine dinucleotide-enriched oligo-deoxynucleotides. The induction of PR domaincontaining protein 1 (PRDM1; also known as Blimp-1), a critical regulator of plasmacytic differentiation, by these agents was closely correlated but varied between individual cases, despite functionally intact IL-21 receptor- and Toll-like receptor 9-mediated signal transducer and activator of transcription 3, and nuclear factor-кB pathways. PRDM1 induction was inversely correlated with the extent of anergy as measured by the ability to mobilize intracellular $\mathrm{Ca}^{2+}$ following $\mathrm{BCR}$ crosslinking. PRDM1 responsiveness was associated with other markers of differentiation and proliferation but not with differences in apoptosis. The ability to induce PRDM1 did correlate with differential transcriptional and epigenetic regulation of the PRDM1 gene. These studies extend our understanding of CLL pathobiology, demonstrating that reduced differentiation capacity may be a consequence of anergy. Epigenetic drugs may offer possibilities to reactivate PRDM1 expression as part of novel differentiation therapy approaches. (Blood. 2014;123(21):3277-3285)

\section{Introduction}

Chronic lymphocytic leukemia (CLL) is a malignancy of B lymphocytes that retain dependency on extracellular stimuli for their survival and behavior. Two major CLL subsets have been identified which arise at distinct stages of B-cell differentiation and are characterized by varying levels of somatic hypermutation of immunoglobulin (Ig) V-genes. ${ }^{1}$ Importantly, these subsets exhibit very different clinical behavior. CLL with unmutated V-genes (U-CLL) appears to develop from naive $B$ cells of the natural antibody repertoire with specificity for common pathogens and has a significantly worse prognosis compared with CLL with mutated V-genes (M-CLL). ${ }^{2-5}$ Further support for a role of antigen stimulation in CLL is provided by the presence of biased $\mathrm{V}$-gene usage in both subsets and the presence of conserved sequence motifs within the complementarity determining region $3 \mathrm{~s}$ of CLL-derived B-cell receptors (BCRs). ${ }^{6-8}$ Putative CLL antigens have been identified, including self-antigens, or antigens derived from common pathogens including viruses, bacteria, and fungi. ${ }^{9}$ The idea that BCR signaling is important in CLL is supported by recent clinical evidence describing dramatic responses following the administration of BCR-associated signaling kinase inhibitors. ${ }^{10}$

The identification of altered surface $\operatorname{IgM}(\mathrm{sIgM})$ glycosylation and "endocytosis in vivo," whereby sIgM expression and signal capacity are variably down-modulated in circulating malignant cells, demonstrates that antigen engagement is on-going in most, if not all, CLL. ${ }^{10,11}$ The major consequence of chronic antigen engagement appears to be the induction of anergy, ${ }^{12,13}$ a mechanism of tolerance in normal B cells whereby autoreactive cells are rendered nonresponsive to activation via their cell surface BCRs due to antigen stimulation in the absence of supporting secondary signals. ${ }^{14}$ CLL anergy is associated with strong down-modulation of sIgM expression and signaling capacity, elevated phosphorylation of extracellular signal-regulated kinases (ERK), and increased activation of nuclear factor of activated T cells (NFAT). ${ }^{12,13}$ Similar to anergy in normal B cells, ${ }^{15}$ CLL anergy is at least partly reversible, as culture of CLL cells in vitro results in recovery of sIgM expression and function, and reduced basal ERK phosphorylation. ${ }^{12,16}$ Anergy is most apparent in M-CLL and is associated with good prognosis. ${ }^{12}$ However, in some cases, possibly low levels of "positive" signaling leading to induction of cell proliferation- and survival-promoting pathways appears to tip the balance of response toward progressive disease. For example, the pro-proliferation protein MYC and the survival protein myeloid cell leukemia sequence 1 (MCL1), which are induced following $\operatorname{sgM}$ stimulation in vitro, are more highly
Submitted November 19, 2013; accepted March 7, 2014. Prepublished online as Blood First Edition paper, March 17, 2014; DOI 10.1182/blood-2013-11539049.
The publication costs of this article were defrayed in part by page charge payment. Therefore, and solely to indicate this fact, this article is hereby marked "advertisement" in accordance with 18 USC section 1734.

The online version of this article contains a data supplement. 
expressed in circulating blood cells in U-CLL compared with M-CLL. ${ }^{17-20}$ In normal B cells, anergy is associated with reduced differentiation in response to a range of stimuli. ${ }^{14}$ This lack of response presumably acts to prevent the production of potentially damaging autoantibodies. Previous studies have shown that CLL cells exhibit varied capacity to undergo differentiation. ${ }^{21-23}$ However, the mechanism that underlies this variation in differentiation capacity and whether this is linked to the anergic phenotype of CLL cells is unknown.

In this study, we have investigated the responses of primary CLL samples to two factors, which can promote differentiation of normal B cells, the cytokine IL-2 $1^{24}$ and cytosine guanine dinucleotideenriched oligo-deoxynucleotides $(\mathrm{CpG}-\mathrm{ODN})^{25}$; the latter acting via the pattern recognition receptor, Toll-like receptor (TLR) $9 .{ }^{26} \mathrm{We}$ demonstrated that CLL anergy, defined by reversible downmodulation of sIgM signaling capacity, is closely associated with reduced differentiation responses to either IL-21 or CpG-ODN. Reduced differentiation capacity, in turn, was associated with transcriptional repression of the PRDM1 gene which encodes PR domain-containing protein 1 (PRDM1; also known as Blimp-1), a major positive regulator of plasma cell (PC) differentiation. ${ }^{27,28}$ These results provide new insight into the biology of CLL and mechanisms of anergy regulation in both normal and malignant $B$ cells.

\section{Patients, materials, and methods}

\section{Cell preparation and culture}

This study had approval from the Liverpool Research Ethics Committee, and CLL cells were isolated from the peripheral blood of consenting donors (see supplemental Table 1 on the Blood Web site). The study was conducted in accordance with the Declaration of Helsinki. Normal B cells were obtained from buffy coats (Liverpool National Blood Service). Cryopreserved CLL cells from the University of Liverpool Leukemia Tissue Bank were thawed and used as previously described. ${ }^{29}$ For CLL samples which comprised $<95 \%$ malignant $\left(\mathrm{CD}^{+} \mathrm{CD}^{+} 9^{+}\right.$) cells and for all normal B preparations, cells were purified (to $>95 \% \mathrm{CD} 5^{+} \mathrm{CD} 19^{+}$cells) using negative selection (MACS system; Miltenyi Biotec). Cells were cultured at $37^{\circ} \mathrm{C}$ with a density of $3 \times 10^{6}$ cells $/ \mathrm{mL}$ in culture media (RPMI-1640 [Sigma-Aldrich] supplemented with $0.5 \%[\mathrm{w} / \mathrm{v}]$ bovine serum albumin, $2 \mathrm{mM}$ L-glutamine, 100 units $/ \mathrm{mL}$ penicillin, and $100 \mu \mathrm{g} / \mathrm{mL}$ streptomycin). IGHV gene analysis was performed as previously described. ${ }^{30}$

\section{Reagents}

The antibodies and primers used in this study are listed in supplemental Table 2. IL2 and IL-21 were from Invitrogen, type B CpG-ODN 2006 was from InvivoGen, and soluble recombinant human CD40 ligand (CD40L) was from Axxora (UK) Ltd. Phorbol-12-myristate-13-acetate (PMA) and ionomycin were from Sigma-Aldrich. Secreted Ig was quantified using human IgM, IgG, or IgA enzyme-linked immunosorbent assay (ELISA) quantification sets (Bethyl Laboratories).

\section{Morphology}

After culture for 5 days, nonapoptotic CLL cells were negatively purified using MACS purification and cytospun. Apoptotic cells were identified using fluorescein isothiocyanate-conjugated Annexin V, and then removed using antifluorescein isothiocyanate beads. Images were obtained using the Nikon ACT-1 v2.63 microscope software at $\times 100$ magnification.

\section{Nuclear factor-кB activation}

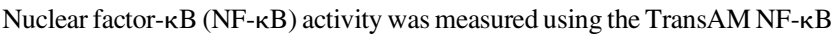
p65 ELISA kit (active motif) and a modified nuclear isolation method. Nuclei were extracted by lysing cells for 5 minutes on ice in phosphate-buffered saline containing $0.5 \% \mathrm{NP}-40,2 \mathrm{mM}$ ethylenediaminetetraacetic acid, and protease inhibitors; $2 \mu \mathrm{g}$ of the extract was added to each ELISA well.

Additional experimental details for analysis of intracellular $\mathrm{Ca}^{2+}\left(\mathrm{iCa}^{2+}\right)$ mobilization, immunoblotting, RNA analysis, culture conditions involving soluble and immobilized CD40L stimulation, and chromatin immunoprecipitation (ChIP) are provided in the supplemental Methods.

\section{Results}

\section{slgM anergy in CLL cells is associated with reduced differentiation capacity}

B-cell anergy is characterized by reduced anti-IgM-induced $\mathrm{iCa}^{2+}$ mobilization in both mouse models ${ }^{14}$ and primary CLL cells. ${ }^{12}$ Therefore, to investigate the potential links between anergy and differentiation capacity, we first characterized anti-IgM-induced $\mathrm{iCa}^{2+}$ mobilization in a cohort of 24 CLL samples (14 U-CLL and 10 M-CLL; supplemental Table 1). As previously described, ${ }^{12}$ $\mathrm{iCa}^{2+}$ response was highly variable between individual samples. The percentage of cells within the malignant clone that were responsive to SIgM stimulation varied from $0 \%$ to $65 \%$, and 17 of 24 samples were considered as signaling responsive, based on the previously defined cutoff of $5 \%$ responding cells. ${ }^{12}$ Thus, our analysis confirmed previous studies that identified variable levels of sIgM anergy in primary CLL samples. Analysis of additional samples obtained from 2 patients at later time points (10 months for case 2 ; 3 months for case 9) confirmed that $\mathrm{iCa}^{2+}$ signaling responses were relatively stable during the course of the disease (supplemental Table 1).

We next investigated the ability of IL-21 to induce differentiation in vitro in these CLL samples. Induction of PRDM1 expression was used as a surrogate marker for the ability of CLL cells to differentiate insofar as this transcriptional repressor is both essential and sufficient for PC differentiation. ${ }^{27,28}$ PRDM1 expression was analyzed using immunoblotting to resolve the PRDM $1 \alpha$ and $\beta$ isoforms, which are generated by alternative promoter usage and are functionally distinct. Whereas PRDM1 $\alpha$, a transcriptional repressor, is a positive regulator of differentiation, repressive functions are absent in PRDM $1 \beta{ }^{31}$ Figure $1 \mathrm{~A}$ shows representative results of PRDM1 immunoblot analysis of unstimulated PC leukemia (PCL) cells, and unmanipulated M-CLL and U-CLL samples. PCL cells were analyzed to confirm the selectivity of the antibody, and as expected, these cells expressed readily detectable levels of both the PRDM $1 \alpha$ and $\beta$ isoforms (97 and $80 \mathrm{kDa}$, respectively), although expression of PRDM1 $\alpha$ was substantially higher than PRDM1 $\beta$. In contrast, unstimulated CLL samples expressed little or no PRDM1 protein. Treatment of CLL cells with IL-21 led to variable induction of PRDM1 protein expression in individual cases. Some cases showed little or no change, whereas others exhibited significant upregulation after stimulation compared with untreated controls. The median induction of PRDM1 $\alpha$ was 2.05 -fold (range $=0.75$ to 5.68; supplemental Figure 2 and supplemental Table 1), and using a twofold increase as a cutoff, 14 of 26 cases were classified as responsive. Figure 1B shows representative results obtained with one IL-21 responsive and one IL-21 nonresponsive sample treated with various concentrations of IL-21. PRDM1 $\alpha$ was induced much more strongly than PRDM1 $\beta$ in responsive samples. Importantly, densitometric quantitation of the immunoblots demonstrated that the fold-induction of PRDM1 $\alpha$ expression, following treatment with IL-21, was positively correlated with the extent of anti-IgM-induced $\mathrm{iCa}^{2+}$ mobilization, regardless of whether PRDM1 responses were 
A

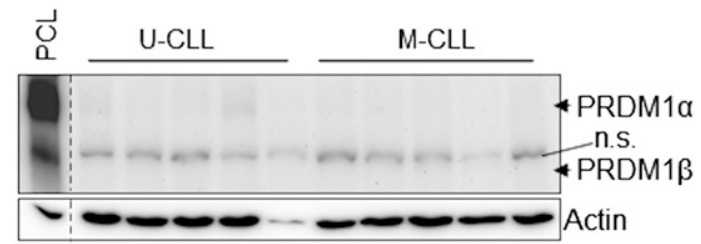

B

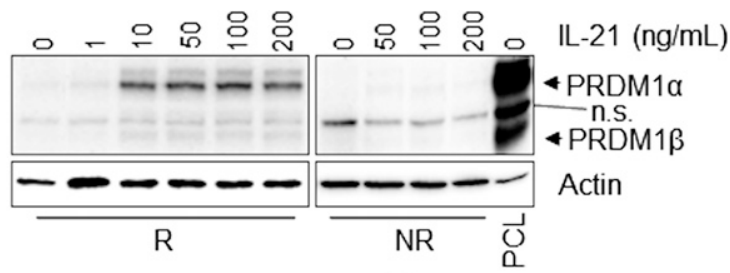

C i)

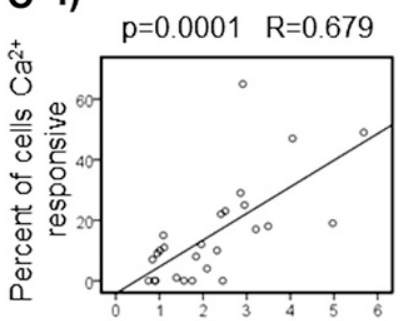

Fold induction of PRDM1

ii)

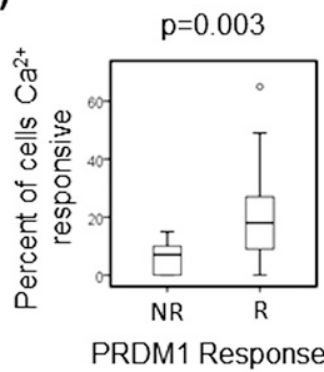

Figure 1. Induction of PRDM1 following treatment with IL-21 in CLL cells correlates with anergy. (A) Basal (ie, unstimulated) levels of PRDM1 protein in M-CLL and U-CLL samples ( $n=5$ each). A PCL sample was used as a positive control. n.s.: nonspecific band detected by the PRDM1 antibody. $\beta$-actin was analyzed as a loading control. (B) PRDM1 induction in representative "responsive" (R) and "non-responsive" (NR) CLL samples after 2 days in culture with the indicated concentrations of IL-21 $(n=3)$. (C) Correlation of PRDM1 $\alpha$ protein induction in cells treated with $50 \mathrm{ng} / \mathrm{mL}$ IL-21 for 16 hours. The maximum percentage of cells that showed increased $\mathrm{iCa}^{2+}$ following anti-lgM stimulation: (i) shows linear correlation between these variables, and (ii) shows significant statistical difference in $\mathrm{iCa}^{2+}$ responsiveness between PRDM1 NR ( $\leq$ twofold induction) and R (>twofold induction) CLL samples $(n=26)$. Fold induction of PRDM1 $\alpha$ was calculated as a ratio by western blot densitometry between the IL-21-treated and untreated samples. The results of statistical evaluation are shown, (i) Pearson's coefficient, and (ii) Mann-Whitney $U$ test.

considered as a continuous variable (Figure $1 \mathrm{Ci} ; P=.0001$ ) or were dichotomized around the twofold cutoff to define samples as "responsive" or "nonresponsive" (Figure 1Cii; $P=.003$ ).

Further experiments were performed to characterize in more detail, the effect of IL-21 on PRDM1 expression in CLL samples. In IL-21 responsive samples, PRDM1 induction was observed within 16 hours following treatment with 10 to $50 \mathrm{ng} / \mathrm{mL}$ of IL-21, although maximal induction was observed after 4 days (Figure 1B and supplemental Figure 1). However, PRDM1 was not induced in nonresponsive CLL samples even in cells that were exposed to substantially higher cytokine concentrations ( $200 \mathrm{ng} / \mathrm{mL}$; Figure 1B) or longer incubation times (supplemental Figure 1). PRDM1 induction was analyzed in an additional 40 samples, so that a total of 66 samples were analyzed following treatment with IL-21 $(50 \mathrm{ng} / \mathrm{mL})$ for 16 hours (supplemental Figure 2). Based on the twofold cutoff, $32 \%$ (21 of 66) of samples were considered to be "responsive" for IL-21-induced PRDM1 expression. Similar to $\mathrm{iCa}^{2+}$ responses, the responsiveness of PRDM1 to IL-21-induced expression was also relatively stable in repeat samples obtained at later disease stages, including the 2 patients analyzed for anti-IgM responses (supplemental Figure 2 and supplemental Table 1).

We also investigated responses to a second differentiation-inducing factor, $\mathrm{CpG}-\mathrm{ODN}$, to determine whether variable differentiation responses were specific to IL-21 or represented a broader variation in differentiation capacity. Thirty-eight CLL samples were cultured for 16 hours with IL-21, CpG-ODN, or left untreated as a control, and PRDM1 induction was analyzed using immunoblotting. Similar to IL-21 responses, induction of PRDM1 following treatment with CpG-ODN was variable between samples (Figure 2A). The maximal induction of PRDM1 was detected in cells treated with 0.1 to $0.5 \mu \mathrm{g} / \mathrm{mL} \mathrm{CpG-ODN} \mathrm{(Figure} \mathrm{2B),} \mathrm{and} \mathrm{in} \mathrm{general,} \mathrm{CpG-ODN} \mathrm{in-}$ duced higher levels of PRDM1 expression compared with IL-21 (Figure 2A). There was a close correlation between the ability of IL-21 and CpG-ODN to induce PRDM1 expression in individual samples $\left(P=3 \times 10^{-6} ; \mathrm{R}=0.66\right)$, and, similar to IL-21, the ability of CpG-ODN to induce PRDM1 expression was associated with the extent of anti-IgM-induced $\mathrm{iCa}^{2+}$ mobilization $(P=.04 ; \mathrm{R}=0.64)$ (Figure 2Ci-ii). Thus, CLL anergy, as defined by lack of sIgM responsiveness, ${ }^{12}$ is associated with reduced differentiation capacity in response to robust PC-inducing stimuli that act by independent signaling pathways.

\section{The heterogeneous effects of IL-21 or CpG-ODN on PRDM1 induction are linked to proliferation but not survival}

Previous studies have demonstrated that IL-21 and CpG-ODN modulate both cell survival and proliferation in CLL cells $\mathrm{s}^{32-35}$ and it was important to probe the relationship between variable effects on PRDM1 induction and these responses. To address this, we selected a cohort of samples that were either responsive or nonresponsive to IL-21/CpG-ODN (based on PRDM1 induction), and investigated the effects of these agents on survival and proliferation.

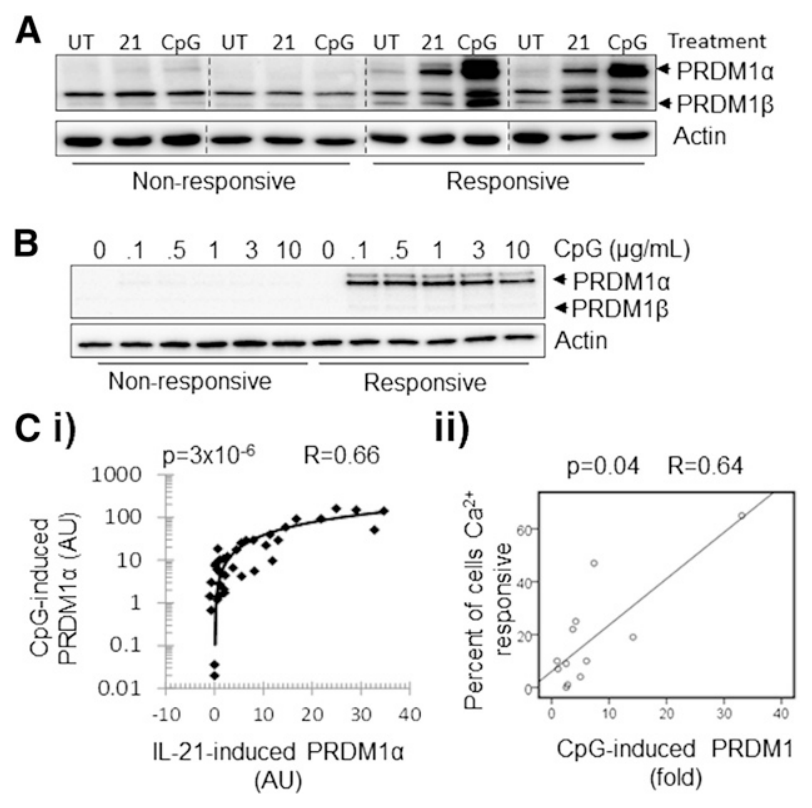

Figure 2. CpG-ODN-mediated induction of PRDM1 is variable and correlates with IL-21 responsiveness in individual CLL cases. (A) Western blot showing levels of PRDM1 protein in representative responsive and nonresponsive CLL clones after being left untreated or following treatment with either IL-21 $(21 ; 50 \mathrm{ng} / \mathrm{mL})$ or CpG-ODN (CpG; $0.5 \mu \mathrm{g} / \mathrm{mL}$ ) for 16 hours $(n=38)$. (B) PRDM1 induction in representative responsive and nonresponsive samples after 2 days in culture with indicated concentrations of CpG-ODN (CpG) ( $n=38)$. (C) PRDM1 induction following 16 hours exposure to CpG-ODN correlates with: (i) PRDM1-induction after 16 hours IL-21 treatment, and (ii) $\mathrm{iCa}^{2+}$ responsiveness following anti-IgM stimulation. Induced PRDM1 $\alpha$ was calculated by subtracting untreated from treated measurements by western blot densitometry. Fold PRDM1 induction was calculated as in Figure $1 \mathrm{C}$. The results of statistical evaluation are shown, (i) Spearman's coefficient, and (ii) Pearson's coefficient. 


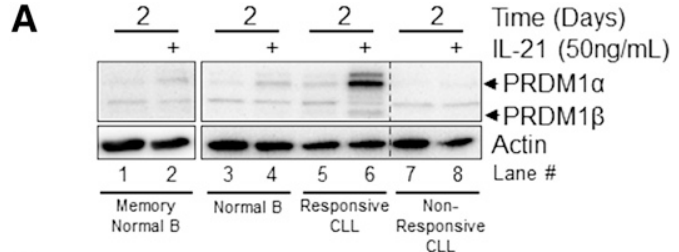

B
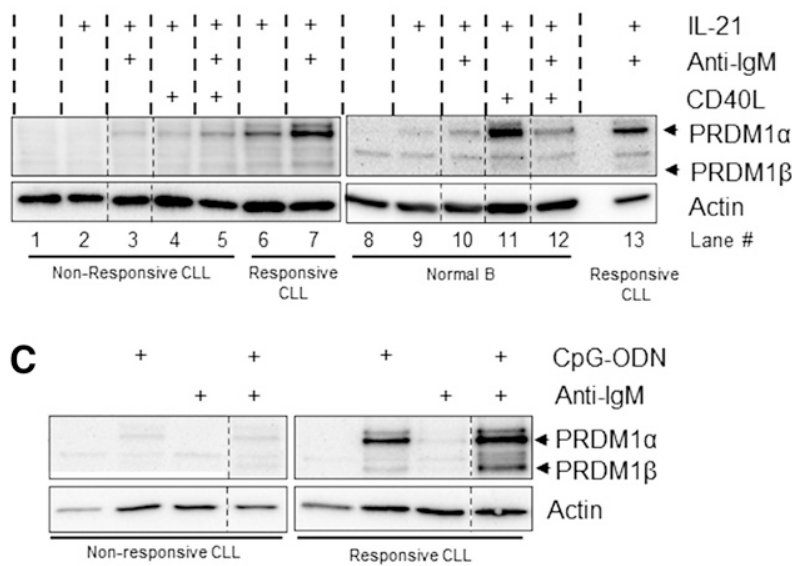

Figure 3. Reduced differentiation capacity in CLL samples is not overcome by additional co-stimuli. (A) Induction of PRDM1 protein in normal B cells $\left(\mathrm{CD} 19^{+} \mathrm{CD} 27^{+}\right.$[memory normal B] and total $\mathrm{CD} 19^{+}$[normal B]) after 2 days of treatment with IL-21, compared with levels seen in representative responsive and nonresponsive CLL samples $(n=3)$. (B) PRDM1 expression in a representative nonresponsive $\mathrm{CLL}$ clone and normal B-cell $\left(\mathrm{CD} 19^{+}\right)$sample after 2 days of treatment with IL-21 \pm anti-IgM \pm CD40L. The lysate of a responsive CLL clone treated for 2 days with IL-21 \pm anti-IgM is included in each western blot for comparison (right hand lanes) $(n=4)$. (C) PRDM1 induced in representative responsive and nonresponsive CLL samples after 2 days of treatment with CpG-ODN \pm anti-lgM $(n=4)$.

Consistent with previous studies, ${ }^{23,32,34}$ we found that the effects of IL-21 on CLL-cell survival in our cohort were highly variable (induction of apoptosis, survival, or no effect). There was no clear relationship between IL-21-induced apoptosis/survival and PRDM1 responsiveness (supplemental Figure 3A-B; $P=.153$ ). Responses to $\mathrm{CpG-ODN}$ were more consistent with enhanced survival in all samples tested, regardless of their responsiveness to CpG-ODNinduced PRDM1 expression ( $P=1.0$ for increase in cell survival in responsive and nonresponsive samples; supplemental Figure 3B). Overall, these results show no clear relationship between PRDM1 inducibility and effects on survival, indicating that these responses are uncoupled. Thus, the failure to induce PRDM1 expression in nonresponding samples cannot simply be a consequence of induction of apoptosis in a subset of samples.

Similar experiments were performed to investigate the effects of IL-21 and CpG-ODN on proliferation in responsive/nonresponsive samples (supplemental Figure 3C). Interestingly, both IL-21 and CpG-ODN promoted higher proliferation rates in PRDM1 responding clones in contrast to nonresponsive cases (IL-21, $P=.02$; CpG-ODN, $P=.05)$.

\section{Co-stimulation does not overcome reduced PRDM1 responses in CLL cells}

Normal B cells require co-stimulation for maximal induction of PRDM1 expression following treatment with IL-21 or CpGODN. ${ }^{24,25} \mathrm{We}$, therefore, determined whether co-stimulation using CD40L and/or anti-IgM overcame the reduced differentiation responses of anergic CLL cells. We compared the responses of CLL cells to those of total $\left(\mathrm{CD} 19^{+}\right)$and memory $\left(\mathrm{CD} 19^{+}\right.$ $\mathrm{CD} 27^{+}$) normal B cells obtained from healthy donors. As previously demonstrated, ${ }^{24}$ IL-21 alone modestly induced PRDM1 in both normal total and memory B cells (Figure 3A). IL-21-induced PRDM1 expression was enhanced by co-stimulation with CD40L or anti-IgM (CD40L > anti-IgM), but the addition of both CD40L and anti-IgM resulted in lower levels of IL-21-induced PRDM1 protein compared with co-stimulation with CD40L alone (Figure 3B, lanes 10-12). Compared with normal B cells, IL-21 alone induced higher levels of PRDM1 expression in responsive CLL samples (Figure 3A, lanes 2, 4, and 6). Co-stimulation with anti-IgM (Figure 3B, lanes 6 and 7) also enhanced IL-21-induced PRDM1 expression in responsive CLL samples, consistent with the retained $\operatorname{IgM}$-responsiveness of these samples $(n=4)$. However, IL-21 nonresponsive CLL cells had considerably less PRDM1 induction upon addition of CD40L or anti-IgM (either alone or in combination) compared with responsive cases (Figure 3B lanes 3-5). Similar results were obtained for both soluble and immobilized CD40L stimulation of CLL samples (not shown). Comparable to IL-21, anti-IgM co-stimulation enhanced CpG-ODN-induced PRDM1 expression in differentiation competent samples, but had little or no effect in nonresponsive samples (Figure 3C).

\section{Reduced PRDM1 induction in anergic CLL cells is reflected by a block in differentiation and Ig secretion}

To confirm that differences in induction of PRDM1 reflected bona fide differences in differentiation, we characterized Ig secretion, surface phenotype, and morphologic changes of CLL cells treated with IL-21 in the presence or absence of co-stimulation. In samples that were competent for induction of PRDM1, we detected increased secretion of IgM (but not IgG or IgA, not shown) following treatment with IL-21 for 5 days, consistent with differentiation (Figure 4A). By contrast, IgM secretion was not increased in samples that were not capable of increasing PRDM1 expression following treatment with
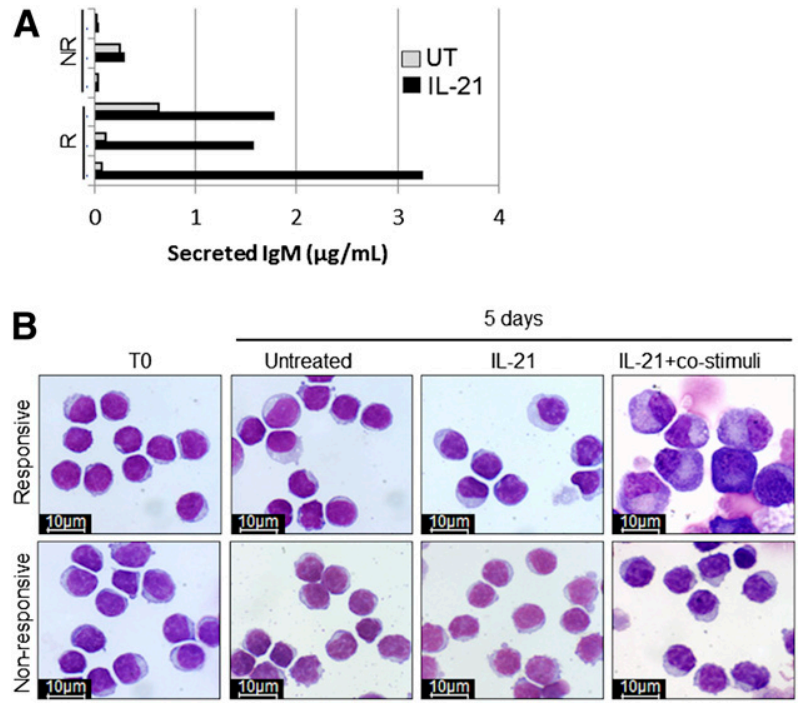

Figure 4. Induction of PRDM1 in responsive CLL samples correlates with increased differentiation. (A) Secreted IgM levels after 5 days of treatment of responsive (R) and nonresponsive (NR) CLL samples with IL-21. (B) Morphology of representative responsive and nonresponsive CLL samples $(n=3$ each) examined directly ex vivo (TO), after 5 days in culture medium alone (untreated), or after 5 days of stimulation by IL-21 with and without co-stimuli (anti-IgM, CD40L, and IL-2) ( $\times 100$ magnification; Nikon ACT-1 v2.63 microscope software Giemsa staining). 
A
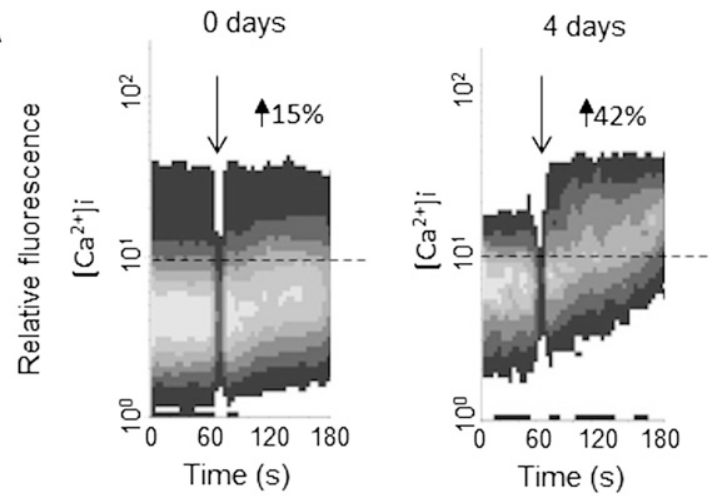

B

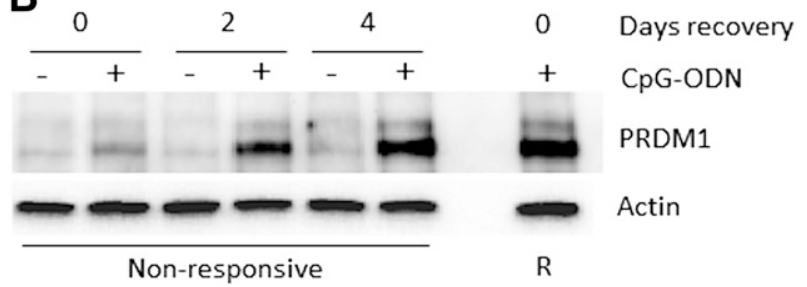

Figure 5. Reversal of anergy following culture in vitro is associated with recovery of PRDM1 induction in nonresponsive CLL samples. Nonresponsive CLL samples $(n=8)$ were left to "recover" in vitro for up to 4 days, and then, $(A)$ $\mathrm{iCa}^{2+}$ mobilization following anti-IgM treatment was measured. The time of anti-lgM stimulation (down arrow) and the set threshold above which the percentage of responsive cells was calculated (dotted line) are shown. (B) Induction of PRDM1 after treatment with CpG-ODN for 16 hours was measured by western blot. Levels of PRDM1 in a responsive CLL clone treated with CpG-ODN for 16 hours are shown for comparison.

IL-21 or CpG-ODN. Despite the production of increased IgM, there were no morphologic changes characteristic of $\mathrm{PC}$ differentiation in CLL cells treated with IL-21 alone (Figure 4B). However, morphologic changes were observed in some samples stimulated with IL-21 plus CD40L, anti-IgM, and IL-2. Like IgM secretion, morphologic changes were observed only in samples that were competent for induction of PRDM1. Despite changes in morphology, CLL cells did not acquire the classical surface PC phenotype (CD38 $8^{\text {high }}, \mathrm{CD} 27^{\text {high }}$, $\left.\mathrm{IgD}^{\text {low }}, \mathrm{CD} 20^{\text {low }}\right)$, whereas this phenotype was observed in approximately one-third of normal B cells following treatment with the same stimuli (supplemental Figure 4). Overall, these results confirm that impaired induction of PRDM1 in CLL samples is associated with reduced differentiation.

\section{Reversal of CLL-cell anergy following culture in vitro is associated with increased PRDM1 responsiveness}

B-cell anergy is antigen dependent and is therefore reversible following the removal of antigen. ${ }^{15}$ In CLL, culture in vitro can result in a reversal of anergy, associated with recovery of sIgM expression and sIgM signaling capacity, although the extent of reversal is variable between individual samples. ${ }^{12}$ To determine if reduced differentiation capacity was also reversible, nonresponsive CLL samples $(\mathrm{n}=8)$ were cultured in vitro for up to 4 days and analyzed for antiIgM-induced $\mathrm{iCa}^{2+}$ mobilization and CpG-ODN-induced PRDM1 expression. Consistent with previous studies, ${ }^{12}$ culture in vitro was associated with variable levels of recovery of anti-IgM signaling responsiveness (Figure 5A). Importantly, samples that showed recovery of sIgM responsiveness following culture in vitro also acquired the ability to induce expression of PRDM1 following treatment with CpG-ODN $(n=5$; Figure 5B). By contrast, those samples that did not recover anti-IgM signaling capacity $(\mathrm{n}=3)$, also did not recover PRDM1 responsiveness. Variability in recovery may reflect different levels of spontaneous apoptosis in CLL cultures, which, if extensive, would preclude functional recovery.

\section{Mechanisms of reduced differentiation capacity in anergic CLL cells}

We performed a series of experiments to determine the mechanisms that caused reduced PRDM1 induction in CLL cells. Analysis of IL-21R expression by flow cytometry demonstrated that IL-21 responsiveness was unrelated to changes in expression of the IL-21 receptor (supplemental Figure 5A), and we therefore focused on downstream signaling responses.

We analyzed two transcription factors that are modulated by IL-21 and CpG-ODN, namely, signal transducer and activator of transcription 3 (STAT3) and NF-кB. STAT3 is important for PC differentiation following treatment with both IL-21 and CpG-ODN, ${ }^{36,37}$ and NF- $\mathrm{BB}$ activity is essential for both IL-21 and CpG-ODN-induced PRDM1 expression in differentiation-competent CLL cells (supplemental Figure 5B). The activation of these transcription factors was analyzed at up to 6 hours poststimulation with IL-21 or CpG-ODN, a time point at which an increase in PRDM1 expression was observed in responsive samples. Increased activation of STAT3 and NF- $\mathrm{B}$ (measured by analysis of STAT3 $\mathrm{Y}^{705}$ phosphorylation $^{38}$ and increased DNA-binding activity of the p65 NF$\kappa \mathrm{B}$ subunit, respectively [Figure 6A-B], increased expression of downstream target genes [MCL1 for STAT3] [Figure 6A], and ІкВ $\alpha$ and $\mathrm{I} \kappa \mathrm{B} \zeta$ for p65 NF-кB [Figure $6 \mathrm{C}$ ]) was observed in all samples, regardless of whether they were differentiation competent.

These data suggested that the mechanism that led to reduced PRDM1 induction acted downstream of activation of proximal signaling events triggered by IL-21 or TLR9. To confirm this, we determined whether reduced differentiation could be circumvented using pharmacologic agents. We treated cells with PMA and/or ionomycin. In responsive CLL samples, PMA alone was sufficient to induce PRDM1 expression, although to a lower extent than CpG-ODN, and this was modestly enhanced by ionomycin (Figure 6D). By contrast, PMA \pm ionomycin did not induce PRDM1 expression in CLL samples that were unable to induce PRDM1 expression following treatment with IL-21 or CpG-ODN.

\section{Lack of PRDM1 induction is associated with altered epigenetic regulation of the PRDM1 gene}

Because variable induction of PRDM1 in different CLL samples was not due to differences in activation of upstream signal transduction pathways, we focused directly on transcriptional control of PRDM1 at the gene level. We treated CLL samples with IL-21 + CD40L or with $\mathrm{CpG}-\mathrm{ODN}$ for up to 4 and 15 hours, respectively, and quantified the level of PRDM1 $\alpha$ messenger RNA (mRNA) by quantitative polymerase chain reaction (Figure 7Ai-ii). Analysis focused on quantifying PRDM1 $\alpha$ mRNA because this encoded the most abundant PRDM1 protein isoform detected in IL-21/CpG-ODN-stimulated cells. Increased levels of PRDMI $\alpha$ mRNA were detected in responsive samples, with levels showing a steady increase following stimulation with IL-21 + CD40L (Figure 7Ai), but a delayed $(>3$ hours) induction following treatment with $\mathrm{CpG}-\mathrm{ODN}$ (Figure 7Aii). In contrast, PRDM1 $\alpha$ mRNA was not induced in nonresponsive samples at any time point. Similar results were obtained when we analyzed expression of "unspliced" (precursor) PRDM1 $\alpha$ RNA in CpG-ODN-treated cells (supplemental Figure 6A). Reduced 


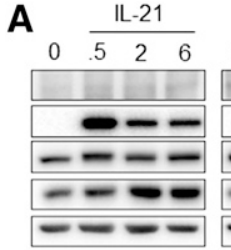

NR-CLL
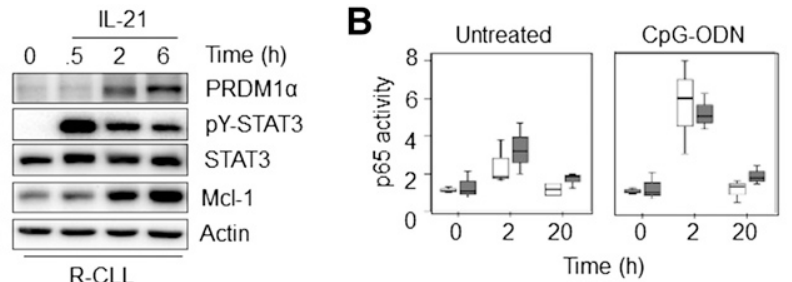

PRDM1 Response

$\square$ NR-CLL $\square R-C L L$
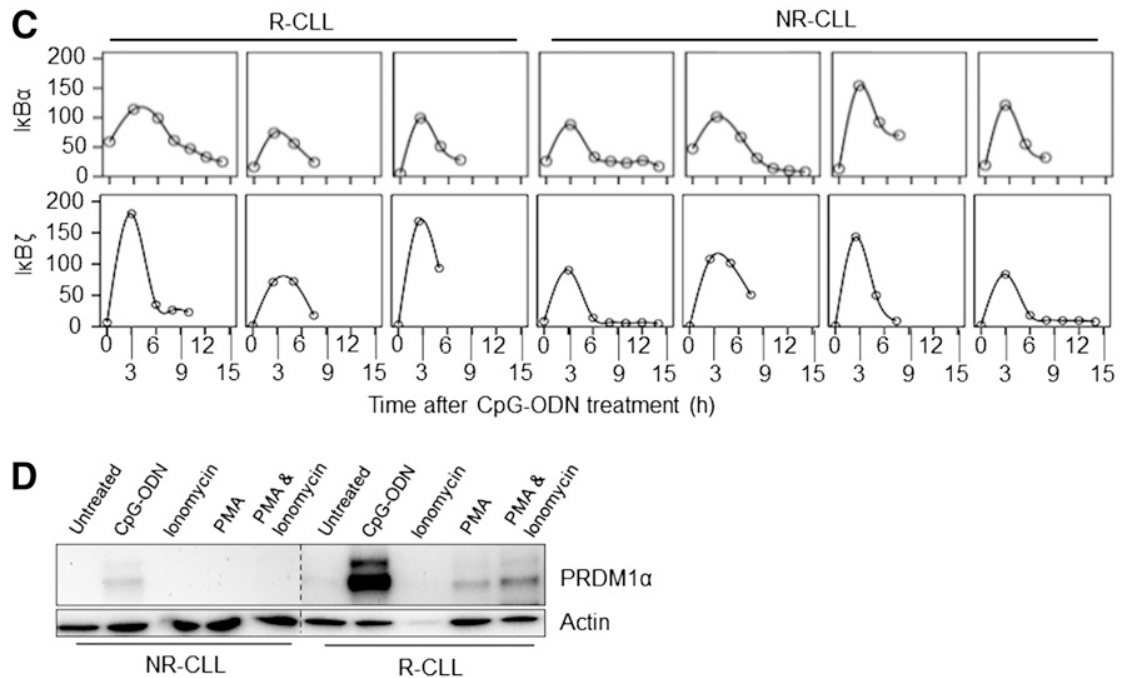

induction of PRDM1 protein is, therefore, likely to be due to pretranscriptional regulatory mechanisms.

We next investigated whether decreased PRDMI transcription was linked to epigenetic mechanisms by investigating both DNA methylation and histone modifications associated with PRDM1 transcriptional control elements. Although PRDMI expression can be

A i)

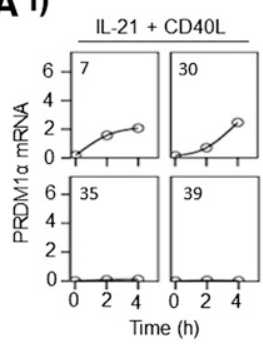

B i)
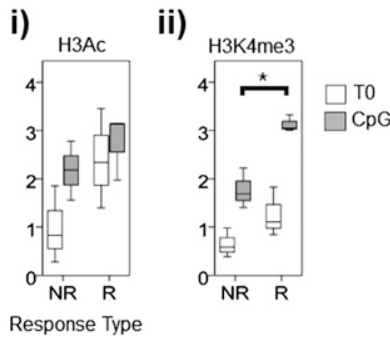

ii)

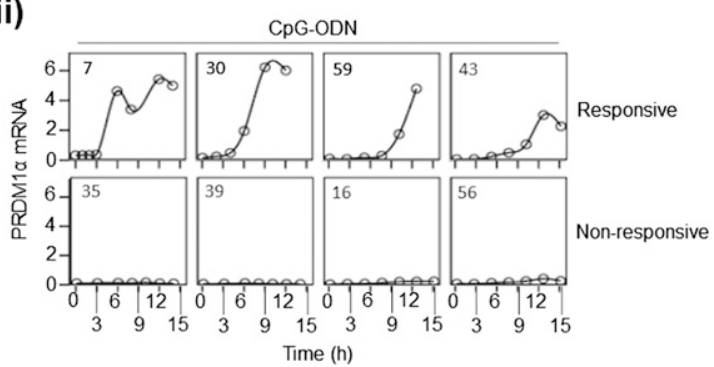

Figure 7. Persistence of PRDM1 $\alpha$ transcriptional repression in nonresponsive CLL samples is a result of failure to induce high levels of activating histone modifications. (A) Responsive and nonresponsive CLL samples were treated with: (i) IL-21 + CD40L, or (ii) CpG-ODN and PRDM1 $\alpha$ RNA was quantified using quantitative polymerase chain reaction at times up to 4 and 15 hours, respectively. Case numbers are shown in the top left-hand corner of each graph. (B) ChIP experiments showing: (i) $\mathrm{H} 3 \mathrm{~K} 9 \mathrm{Ac} / \mathrm{K} 14 \mathrm{Ac}(\mathrm{H} 3 \mathrm{Ac})$, or (ii) $\mathrm{H} 3 \mathrm{~K} 4 \mathrm{me} 3$ at the PRDM1 TSS before and after treatment of responsive $(R ; n=3)$ and nonresponsive $(N R ; n=3)$ samples with CpG-ODN. ${ }^{\star} P=.009$. inhibited by DNA methylation at its transcription start site (TSS), ${ }^{39}$ we detected no evidence for this modification in 3 responsive and 3 nonresponsive samples (a total of $41 \mathrm{CpG}$ sequences analyzed between $-349 \mathrm{bp}$ and $+251 \mathrm{bp}$ from the TSS for each sample; data not shown). To investigate if the variability in PRDM1 expression was due to epigenetic reprogramming, we performed ChIP analysis and observed that chromatin marks associated with active transcription, namely, H3K9/K14 acetylation or H3K4 trimethylation ${ }^{40,41}$ were higher at the TSS in responsive CLL samples compared with nonresponsive samples (Figure 7Bi-ii). Abundance of these marks increased following treatment with $\mathrm{CpG}-\mathrm{ODN}$ in both responsive and nonresponsive CLL cells, but the increase in $\mathrm{H} 3 \mathrm{~K} 4 \mathrm{me} 3$ was significantly higher in responsive samples $(P=.009)$.

Thus, the inability of nonresponsive CLL cells to induce PRDM1 is likely the result of transcriptional repression and failure to facilitate gene transcription through appropriate chromatin modifications. Occupancy of the proximal promoter by appropriately modified RNA polymerase II dictates transcription as "paused" or "poised". ${ }^{42}$ To ascertain the status of the PRDM1 $\alpha$ gene, we performed a ChIP analysis and demonstrated that the promoter is "poised," and initiated for transcription in both response types, as characterized by similar binding of Ser5-phosphorylated RNA polymerase II to its TSS $^{43}$ (supplemental Figure 6B). As further evidence of the "poised" state, H3K27me3 at the TSS was similar in all cases studied (not shown). To conclude, the transcriptional inactivity of the PRDMI gene is due to transcriptional "repression" and not silencing.

\section{Discussion}

Signaling via the BCR is considered to play a major role in determining variable clinical behavior in CLL, a conclusion that is 
consistent with the dramatic clinical responses that have been reported for novel compounds that target BCR-associated kinases. The major consequence of antigen engagement in CLL appears to be anergy, which is observed to a variable extent in all CLL samples. ${ }^{1}$ However, potentially low levels of positive signaling may tip the balance toward cell-survival and division, and disease progression. ${ }^{16,17,44}$ In mouse B cells, anergy is associated with reduced differentiation capacity to limit the production of potentially pathogenic autoantibodies. ${ }^{14}$ Recent studies of potential anergic B-cell populations in healthy humans have demonstrated that these cells are also hyporesponsive to differentiation-promoting signals. ${ }^{45}$ Differentiation capacity of CLL cells is varied, ${ }^{21,22,46-50}$ although it is not known whether this is related to anergy in these cells.

In this study, we demonstrated that variable differentiation capacity of CLL cells is linked to their anergic phenotype with strong anergy associated with markedly reduced differentiation capacity. Anergy is dependent on continued antigen engagement of BCRs, and like downmodulated $\operatorname{sgM},{ }^{12}$ reduced differentiation capacity is reversed following culture in vitro. Our molecular studies revealed that decreased differentiation capacity was associated with transcriptional repression of PRDM1 expression. This transcriptional repression appeared to involve epigenetic reprogramming, a novel observation with potential relevance for understanding B-cell anergy not just in CLL, but also in nonmalignant cells. Given the key role of PRDM1 in controlling differentiation, and as a common node for reception of diverse differentiation-promoting transduction pathways, epigenetic suppression of PRDM1 transcription would be an effective mechanism to broadly and selectively suppress differentiation.

Although IL-21 and CpG-ODN have profound effects on normal B-cell differentiation, their effects on CLL-cell differentiation have not been investigated in detail. One recent study also demonstrated intrasample variation in $\mathrm{CpG}-\mathrm{ODN}$-induced differentiation of CLL samples. ${ }^{23}$ Although not shown in the study, the authors stated that there was a trend to increased $\mathrm{CpG-ODN-induced} \mathrm{differentiation} \mathrm{in}$ U-CLL, which could reflect the impact of anergy that we describe here because anergic features are most prominent in M-CLL. One other study stated that IL-21 did not induce PRDM1 expression in CLL cells, but as this particular component of the work was restricted to just two samples, this could represent the subset of nonresponsive samples identified in this work. ${ }^{32}$

Although our study focused on differentiation, it was important to discover whether variable responses were specific for differentiation, or could be linked to, or is a consequence of, alterations in survival and proliferation. The published literature on proliferative and apoptotic responses of CLL cells following treatment with IL-21 or CpG-ODN is complex. Some studies have demonstrated that IL-21 promotes apoptosis in a proportion of CLL samples, although this response has not been linked to specific subsets of disease. ${ }^{33,35,51} \mathrm{By}$ contrast, other studies have described variable effects on proliferation, with little effect on cell death, potentially linked to $I G H V$ status (enhanced IL-21-induced proliferation in M-CLL). ${ }^{32}$ Variation has also been described for CpG-ODN responses. ${ }^{34}$ To investigate the relationship between these responses and differentiation, we analyzed the effects of IL-21/CpG-ODN on survival and proliferation in a defined cohort of samples with distinct PRDM1 responsiveness. Consistent with the literature, effects of IL-21 on apoptosis were variable, whereas CpG-ODN consistently promoted survival. However, the key observation was that variation in apoptosis responses was clearly unrelated to PRDM1 induction, indicating that these responses are uncoupled. In contrast, responsive CLL cases were significantly more likely to exhibit a proliferative phenotype. Repression of both IL-21/ CpG-ODN-induced proliferation and differentiation in anergic CLL cells may not be surprising insofar as both proliferation and differentiation of $\mathrm{B}$ cells are required for antibody responses.

The role of transcription in B-cell anergy has been a subject of debate. It is clear that B-cell anergy is associated with changes in gene expression compared with naive or acutely stimulated cells, ${ }^{52}$ and transcription factors such as nuclear factor of activated T cells 1 play essential roles in maintaining anergy. ${ }^{53}$ However, some features of anergy are rapidly reversible following removal of the anergizing antigen. ${ }^{15}$ The kinetics of this response would appear to preclude a role for transcriptional reprogramming, at least in modulation of initial sIgM signaling capacity. However, it is possible that other components of the anergic phenotype do depend on transcriptional regulation. In anergic T cells, silencing of the IL2 and IFNG loci is associated with epigenetic reprogramming through changes in histone acetylation and methylation. ${ }^{54}$ Regardless, further studies of CLL cells may shed new light on molecular mechanisms of anergy with relevance not just for the biology of normal and malignant $\mathrm{B}$ cells, but also for autoimmune disorders characterized by a breakdown in B-cell tolerance.

Overall, these studies extend our understanding of the consequences of antigen engagement in CLL and demonstrate that reduced differentiation capacity of CLL cells is an additional feature linked to the anergic phenotype of these cells. Further study is required to explore the in vivo relevance of our findings. Since all CLL samples show some evidence for anergy, our observations may explain the lack of robust differentiation of CLL cells in vivo despite recent evidence for IL-21 and autologous T-cell-induced proliferative signals. ${ }^{32,55}$ However, there is some evidence for low levels of differentiation in some patients, revealed by low level PRDM1 expression in a small subset of lymph nodes from patients with CLL/small lymphocytic lymphoma. ${ }^{56}$ Moreover, CLL cells are known to secrete variable levels of $\operatorname{IgM}^{1}$ and paraproteins are detected in some patients. The clinical significance of these features remain unclear, but it will be interesting to determine whether they are more frequent in the less anergized U-CLL subset. Differentiation-promoting therapies are well established in acute leukemias and have been suggested as a potential strategy for CLL. ${ }^{23,57,58}$ Because CLL cells do appear to retain a latent potential for differentiation, and our results indicate that the PRDMI locus is transcriptionally "poised," it is likely amenable to derepression to promote terminal differentiation and apoptosis. Our results are important in this regard because the residual differentiation capacity appears to rest within the less anergized subsets of disease, which generally have a poorer prognosis, and where there is a pressing need for new or adjuvant treatment approaches.

\section{Acknowledgments}

The authors thank John C. Cawley and Freda K. Stevenson for critical reading of the manuscript and support for the project.

A.D. was supported by the Carol Shepherd Fund. Additional funding for this study was provided by the Royal Liverpool University Hospital Leukemia Fund, Isle of Man Anti-Cancer Fund, and Leukemia and Lymphoma Research, United Kingdom.

\section{Authorship}

Contribution: A.D. designed and performed research, collected, analyzed and interpreted data, performed statistical analysis, and wrote the manuscript; M.G. performed research, analyzed and 
interpreted data, and reviewed the manuscript; J.R.S. designed research, interpreted the data, and wrote the manuscript; G.P. designed research, interpreted data, wrote and edited the manuscript; and N.K. designed research, analyzed and interpreted data, and wrote and edited the manuscript.
Conflict-of-interest disclosure: The authors declare no competing financial interests.

Correspondence: Nagesh Kalakonda, Haematology, $2^{\text {nd }}$ Floor, Duncan Building, Daulby St, Liverpool L69 3GA, United Kingdom; e-mail: nageshk@liv.ac.uk.

\section{References}

1. Stevenson FK, Krysov S, Davies AJ, Steele AJ Packham G. B-cell receptor signaling in chronic lymphocytic leukemia. Blood. 2011;118(16): 4313-4320.

2. Damle RN, Wasil T, Fais F, et al. Ig V gene mutation status and CD38 expression as novel prognostic indicators in chronic lymphocytic leukemia. Blood. 1999;94(6):1840-1847.

3. Forconi F, Potter KN, Wheatley I, et al. The normal IGHV1-69-derived B-cell repertoire contains stereotypic patterns characteristic of unmutated CLL. Blood. 2010;115(1):71-77.

4. Hamblin TJ, Davis Z, Gardiner A, Oscier DG, Stevenson FK. Unmutated Ig $\mathrm{V}(\mathrm{H})$ genes are associated with a more aggressive form of chronic lymphocytic leukemia. Blood. 1999;94(6): 1848-1854.

5. Seiffert M, Dietrich S, Jethwa A, Glimm H, Lichter $\mathrm{P}$, Zenz T. Exploiting biological diversity and genomic aberrations in chronic lymphocytic leukemia. Leuk Lymphoma. 2012;53(6): 1023-1031.

6. Fais F, Ghiotto F, Hashimoto S, et al. Chronic lymphocytic leukemia $B$ cells express restricted sets of mutated and unmutated antigen receptors. J Clin Invest. 1998;102(8):1515-1525.

7. Murray F, Darzentas N, Hadzidimitriou A, et al. Stereotyped patterns of somatic hypermutation in subsets of patients with chronic lymphocytic leukemia: implications for the role of antigen selection in leukemogenesis. Blood. 2008;111(3): 1524-1533.

8. Stamatopoulos K, Belessi C, Moreno C, et al. Over $20 \%$ of patients with chronic lymphocytic leukemia carry stereotyped receptors: pathogenetic implications and clinical correlations. Blood. 2007;109(1):259-270.

9. Rosén A, Murray F, Evaldsson C, Rosenquist R. Antigens in chronic lymphocytic leukemia-implications for cell origin and leukemogenesis. Semin Cancer Biol. 2010;20(6): 400-409.

10. Woyach JA, Johnson AJ, Byrd JC. The B-cell receptor signaling pathway as a therapeutic target in CLL. Blood. 2012;120(6):1175-1184.

11. Krysov S, Potter KN, Mockridge $\mathrm{Cl}$, et al. Surface IgM of CLL cells displays unusual glycans indicative of engagement of antigen in vivo. Blood. 2010;115(21):4198-4205

12. Mockridge $\mathrm{Cl}$, Potter KN, Wheatley I, Neville LA, Packham G, Stevenson FK. Reversible anergy of slgM-mediated signaling in the two subsets of CLL defined by VH-gene mutational status. Blood. 2007;109(10):4424-4431.

13. Muzio M, Apollonio B, Scielzo C, et al. Constitutive activation of distinct BCR-signaling pathways in a subset of CLL patients: a molecular signature of anergy. Blood. 2008;112(1):188-195.

14. Cambier JC, Gauld SB, Merrell KT, Vilen BJ. Bcell anergy: from transgenic models to naturally occurring anergic B cells? Nat Rev Immunol. 2007;7(8):633-643.

15. Gauld SB, Benschop RJ, Merrell KT, Cambier JC Maintenance of $B$ cell anergy requires constant antigen receptor occupancy and signaling. Nat Immunol. 2005;6(11):1160-1167.

16. Apollonio B, Scielzo C, Bertilaccio MT, et al. Targeting B-cell anergy in chronic lymphocytic leukemia. Blood. 2013;121(19):3879-3888, S1-8.
17. Krysov S, Dias S, Paterson A, et al. Surface IgM stimulation induces MEK1/2-dependent MYC expression in chronic lymphocytic leukemia cells. Blood. 2012;119(1):170-179.

18. Pepper C, Lin TT, Pratt G, et al. Mcl-1 expression has in vitro and in vivo significance in chronic lymphocytic leukemia and is associated with other poor prognostic markers. Blood. 2008;112(9): 3807-3817.

19. Petlickovski A, Laurenti L, Li X, et al. Sustained signaling through the B-cell receptor induces Mcl-1 and promotes survival of chronic lymphocytic leukemia B cells. Blood. 2005;105(12): 4820-4827.

20. Zhang W, Kater AP, Widhopf GF II, et al. B-cell activating factor and $v-M y c$ myelocytomatosis viral oncogene homolog (c-Myc) influence progression of chronic lymphocytic leukemia. Proc Natl Acad Sci USA. 2010;107(44): 18956-18960.

21. Drexler HG, Gignac SM, Jones RA, Scott CS, Pettit GR, Hoffbrand AV. Bryostatin 1 induces differentiation of B-chronic lymphocytic leukemia cells. Blood. 1989;74(5):1747-1757.

22. Fluckiger AC, Rossi JF, Bussel A, Bryon P, Banchereau J, Defrance T. Responsiveness of chronic lymphocytic leukemia B cells activated via surface Igs or CD40 to B-cell tropic factors. Blood. 1992;80(12):3173-3181.

23. Gutierrez A Jr, Arendt BK, Tschumper RC, Kay $\mathrm{NE}$, Zent CS, Jelinek DF. Differentiation of chronic lymphocytic leukemia B cells into immunoglobulin secreting cells decreases LEF-1 expression. PLOS ONE. 2011;6(10):e26056.

24. Ettinger R, Sims GP, Fairhurst AM, et al. IL-21 induces differentiation of human naive and memory $\mathrm{B}$ cells into antibody-secreting plasma cells. J Immunol. 2005;175(12):7867-7879.

25. Krieg AM, Yi AK, Matson S, et al. CpG motifs in bacterial DNA trigger direct B-cell activation. Nature. 1995;374(6522):546-549.

26. Hemmi H, Takeuchi O, Kawai $\mathrm{T}$, et al. A toll-like receptor recognizes bacterial DNA. Nature. 2000; 408(6813):740-745.

27. Shapiro-Shelef M, Lin KI, McHeyzer-Williams LJ, Liao J, McHeyzer-Williams MG, Calame K. Blimp1 is required for the formation of immunoglobulin secreting plasma cells and pre-plasma memory B cells. Immunity. 2003;19(4):607-620.

28. Turner CA Jr, Mack DH, Davis MM. Blimp-1, a novel zinc finger-containing protein that can drive the maturation of $B$ lymphocytes into immunoglobulin-secreting cells. Cell. 1994;77(2): 297-306.

29. Lin K, Glenn MA, Harris RJ, et al. c-Abl expression in chronic lymphocytic leukemia cells: clinical and therapeutic implications. Cancer Res. 2006;66(15):7801-7809.

30. Lin K, Sherrington PD, Dennis M, Matrai Z, Cawley JC, Pettitt AR. Relationship between p53 dysfunction, CD38 expression, and $\lg \mathrm{V}(\mathrm{H})$ mutation in chronic lymphocytic leukemia. Blood. 2002;100(4):1404-1409.

31. Györy I, Fejér G, Ghosh N, Seto E, Wright KL. Identification of a functionally impaired positive regulatory domain I binding factor 1 transcription repressor in myeloma cell lines. J Immunol. 2003; 170(6):3125-3133.
32. Ahearne MJ, Willimott S, Piñon L, et al. Enhancement of CD154/IL4 proliferation by the T follicular helper (Tfh) cytokine, IL21 and increased numbers of circulating cells resembling Tfh cells in chronic lymphocytic leukaemia. Br J Haematol. 2013;162(3):360-370.

33. de Totero D, Meazza R, Capaia M, et al. The opposite effects of IL-15 and IL-21 on CLL B cells correlate with differential activation of the JAK/ STAT and ERK1/2 pathways. Blood. 2008;111(2): 517-524.

34. Ghalamfarsa G, Jadidi-Niaragh F, Hojjat-Farsangi $M$, et al. Differential regulation of $B$-cell proliferation by IL21 in different subsets of chronic lymphocytic leukemia. Cytokine. 2013;62(3): 439-445.

35. Gowda A, Roda J, Hussain SR, et al. IL-21 mediates apoptosis through up-regulation of the $\mathrm{BH} 3$ family member BIM and enhances both direct and antibody-dependent cellular cytotoxicity in primary chronic lymphocytic leukemia cells in vitro. Blood. 2008;111(9):4723-4730.

36. Avery DT, Deenick EK, Ma CS, et al. B cellintrinsic signaling through IL-21 receptor and STAT3 is required for establishing long-lived antibody responses in humans. J Exp Med. 2010; 207(1):155-171.

37. Jabara $\mathrm{HH}, \mathrm{McD}$ onald DR, Janssen E, et al DOCK8 functions as an adaptor that links TLRMyD88 signaling to B cell activation. Nat Immunol. 2012;13(6):612-620.

38. Zhong Z, Wen Z, Darnell JE Jr. Stat3: a STAT family member activated by tyrosine phosphorylation in response to epidermal growth factor and interleukin-6. Science. 1994; 264(5155):95-98

39. Iqbal J, Kucuk C, Deleeuw RJ, et al. Genomic analyses reveal global functional alterations that promote tumor growth and novel tumor suppressor genes in natural killer-cell malignancies. Leukemia. 2009;23(6):1139-1151.

40. Santos-Rosa H, Schneider R, Bannister AJ, et al. Active genes are tri-methylated at K4 of histone H3. Nature. 2002;419(6905):407-411.

41. Schübeler D, MacAlpine DM, Scalzo D, et al. The histone modification pattern of active genes revealed through genome-wide chromatin analysis of a higher eukaryote. Genes Dev. 2004 18(11):1263-1271.

42. Brookes E, Pombo A. Modifications of RNA polymerase II are pivotal in regulating gene expression states. EMBO Rep. 2009;10(11) 1213-1219.

43. Buratowski S. Progression through the RNA polymerase II CTD cycle. Mol Cell. 2009;36(4): 541-546.

44. Le Roy C, Deglesne PA, Chevallier N, et al. The degree of BCR and NFAT activation predicts clinical outcomes in chronic lymphocytic leukemia. Blood. 2012;120(2):356-365.

45. Quách TD, Manjarrez-Orduño N, Adlowitz DG, et al. Anergic responses characterize a large fraction of human autoreactive naive $B$ cells expressing low levels of surface IgM. J Immunol. 2011;186(8):4640-4648.

46. Efremidis AP, Haubenstock $\mathrm{H}$, Holland JF, Bekesi JG. Tpa-induced maturation in secretory human B-leukemic cells in vitro: DNA synthesis, antigenic 
changes, and immunoglobulin secretion. Blood. 1985;66(4):953-960.

47. Johnstone AP, Jensenius JC, Millard RE, Hudson L. Mitogen-stimulated immunoglobulin production by chronic lymphocytic leukaemic lymphocytes. Clin Exp Immunol. 1982;47(3):697-705.

48. Okamura J, Letarte M, Stein LD, Sigal NH, Gelfand EW. Modulation of chronic lymphocytic leukemia cells by phorbol ester: increase in la expression, IgM secretion and MLR stimulatory capacity. J Immunol. 1982;128(5):2276-2280.

49. Tretter T, Schuler M, Schneller F, et al. Direct cellular interaction with activated CD4(+) T cells overcomes hyporesponsiveness of B-cell chronic lymphocytic leukemia in vitro. Cell Immunol. 1998; 189(1):41-50.

50. Zupo S, Massara R, Dono M, et al. Apoptosis or plasma cell differentiation of CD38-positive
B-chronic lymphocytic leukemia cells induced by cross-linking of surface IgM or IgD. Blood. 2000; 95(4):1199-1206.

51. Jahrsdörfer B, Blackwell SE, Wooldridge JE, et al. B-chronic lymphocytic leukemia cells and other $B$ cells can produce granzyme $B$ and gain cytotoxic potential after interleukin-21-based activation. Blood. 2006;108(8):2712-2719.

52. Glynne R, Akkaraju S, Healy JI, Rayner J, Goodnow CC, Mack DH. How self-tolerance and the immunosuppressive drug FK506 prevent Bcell mitogenesis. Nature. 2000;403(6770): 672-676.

53. Barrington RA, Borde M, Rao A, Carroll MC. Involvement of NFAT1 in B cell self-tolerance. $J$ Immunol. 2006;177(3):1510-1515.

54. Wells AD. New insights into the molecular basis of T cell anergy: anergy factors, avoidance sensors, and epigenetic imprinting. $J$ Immunol. 2009; 182(12):7331-7341.

55. Pascutti MF, Jak M, Tromp JM, et al. IL-21 and CD40L signals from autologous $T$ cells can induce antigen-independent proliferation of CLL cells. Blood. 2013;122(17): 3010-3019.

56. Garcia JF, Roncador G, García JF, et al. PRDM1/BLIMP-1 expression in multiple B and T-cell lymphoma. Haematologica. 2006;91(4): 467-474.

57. Meacham CE, Morrison SJ. Tumour heterogeneity and cancer cell plasticity. Nature. 2013;501(7467):328-337.

58. Nowak D, Stewart D, Koeffler HP. Differentiation therapy of leukemia: 3 decades of development. Blood. 2009;113(16):3655-3665. 


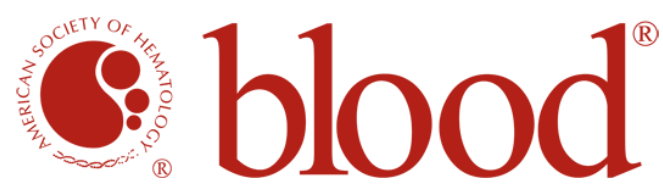

2014 123: 3277-3285

doi:10.1182/blood-2013-11-539049 originally published online March 17, 2014

\section{Variable induction of PRDM1 and differentiation in chronic lymphocytic leukemia is associated with anergy}

Andrew Duckworth, Mark Glenn, Joseph R. Slupsky, Graham Packham and Nagesh Kalakonda

Updated information and services can be found at:

http://www.bloodjournal.org/content/123/21/3277.full.html

Articles on similar topics can be found in the following Blood collections

Lymphoid Neoplasia (2254 articles)

Information about reproducing this article in parts or in its entirety may be found online at:

http://www.bloodjournal.org/site/misc/rights.xhtml\#repub_requests

Information about ordering reprints may be found online at:

http://www.bloodjournal.org/site/misc/rights.xhtml\#reprints

Information about subscriptions and ASH membership may be found online at:

http://www.bloodjournal.org/site/subscriptions/index.xhtml

Blood (print ISSN 0006-4971, online ISSN 1528-0020), is published weekly by the American Society of Hematology, 2021 L St, NW, Suite 900, Washington DC 20036.

Copyright 2011 by The American Society of Hematology; all rights reserved. 